\title{
Renal function is impaired in normotensive chronic HCV patients: role of insulin resistance
}

\author{
Angela Sciacqua $^{1} \cdot$ Maria Perticone $^{2} \cdot$ Eliezer J. Tassone $^{1} \cdot$ Antonio Cimellaro $^{1} \cdot$ \\ Benedetto Caroleo $^{1} \cdot$ Sofia Miceli $^{1} \cdot$ Michele Andreucci $^{3} \cdot$ Anna Licata $^{4}$. \\ Giorgio Sesti ${ }^{1}$. Francesco Perticone ${ }^{1}$
}

Received: 31 July 2015/ Accepted: 28 October 2015

(c) SIMI 2015

\begin{abstract}
Renal dysfunction is an independent predictor for cardiovascular morbidity and mortality. We investigated whether chronic hepatitis $\mathrm{C}$ virus (HCV) infection and the related insulin resistance/hyperinsulinemia influence renal function in comparison with a group of healthy subjects and with another group with metabolic syndrome. We enrolled 130 newly diagnosed HCV outpatients matched for age and gender with 130 patients with metabolic syndrome and 130 healthy subjects. Renal function was evaluated by calculation of glomerular filtration rate (eGFR, $\mathrm{mL} / \mathrm{min} / 1.73 \mathrm{~m}^{2}$ ) using the CKD-EPI equation. The following laboratory parameters were measured: fasting plasma glucose and insulin, total, LDL- and HDL-cholesterol, triglyceride, creatinine, and HOMA to evaluate insulin sensitivity. HCV patients with respect to both healthy subjects and metabolic syndrome patients have a decreased e-GFR: $86.6 \pm 16.1$ vs $120.2 \pm 23.1 \mathrm{~mL} / \mathrm{min} /$ $1.73 \mathrm{~m}^{2}(P<0.0001)$ and $94.9 \pm 22.6 \mathrm{~mL} / \mathrm{min} / 1.73 \mathrm{~m}^{2}$ $(P=0.003)$, respectively. Regarding biochemical
\end{abstract}

A. Sciacqua and M. Perticone equally contributed to the work.

Francesco Perticone

perticone@unicz.it

1 Department of Medical and Surgical Sciences, University Magna Græcia of Catanzaro, V.le Europa, 88100 Catanzaro, Italy

2 Department of Experimental and Clinical Medicine, University Magna Græcia of Catanzaro, Catanzaro, Italy

3 Department of Health Sciences, University Magna Græcia of Catanzaro, Catanzaro, Italy

4 Biomedical Department of Internal and Specialistic Medicine, University of Palermo, Palermo, Italy variables, HCV patients, in comparison with healthy subjects, have a higher triglyceride level, creatinine, fasting insulin and HOMA $(3.4 \pm 1.4$ vs $2.6 \pm 1.3 ; P<0.0001)$. At linear regression analysis, the correlation between e-GFR and HOMA is similar in the metabolic syndrome $(r=-0.555, \quad P<0.0001) \quad$ and $\mathrm{HCV} \quad(r=-0.527$, $P<0.0001)$ groups. At multiple regression analysis, HOMA is the major determinant of e-GFR in both groups, accounting for, respectively, 30.8 and $27.8 \%$ of its variation in the metabolic syndrome and HCV. In conclusion, we demonstrate that HCV patients have a significant reduction of e-GFR and that insulin resistance is the major predictor of renal dysfunction.

Keywords Chronic C hepatitis - Renal function · Insulin resistance $\cdot$ Cardiovascular risk $\cdot$ Metabolic syndrome

\section{Introduction}

Chronic hepatitis $\mathrm{C}$ virus (HCV) infection is associated with several metabolic alterations that contribute to development of subclinical cardiovascular disease, particularly atherosclerotic vascular damage [1-4]. In accordance with this, we recently demonstrate that hyperinsulinemia HCV-related is associated with both increased cardiac mass and arterial vascular stiffness, that are considered independent and strong predictors of cardiovascular events also in different settings of patients [58].

In addition, the metabolic syndrome (MS), characterized by visceral obesity and insulin resistance (IR), is associated with a decrease in renal function that also represents an independent and strong predictor of cardiovascular morbidity and mortality in the general population $[9,10]$. In 
particular, the National Health and Nutrition Examination Survey (NHANES) III, demonstrates a strong association between the MS and the development of chronic kidney disease (CKD); while, data reported by the Atherosclerosis Risk in Communities Study (ARIC) demonstrates a strong correlation between IR status and loss of renal function [11, 12].

On the other hand, there is conflicting evidence about the possible association between HCV infection and CKD; particularly, the majority of these studies fail to show an association between these two clinical conditions [13-16]. Taken together, the aim of this study was to evaluate the possible association between chronic HCV infection-related IR and renal dysfunction in comparison with both healthy subjects and MS patients.

\section{Methods}

\section{Study population}

For this aim, we designed a cross-sectional study involving patients evaluated at the University Hospital of Catanzaro. We recruited $130 \mathrm{HCV}^{+}$normotensive Caucasian outpatients (75 males and 55 females, mean age $55.2 \pm 13.9$ years). They were matched for age and gender in a 1:1:1 ratio with 260 subjects participating in the CAtanzaro MEtabolic RIsk factors Study (CATAMERIS), with 130 patients with MS (mean age $54.6 \pm 10.3$ years) evaluated according NCEP-ATP III criteria [17] and 130 healthy subjects (mean age $54.4 \pm 11.9$ years) [18]. At the time of the first evaluation, both $\mathrm{HCV}^{+}$and MS patients were newly diagnosed and untreated with antiviral therapy or other drugs. In the whole study population, exclusion criteria were type 2 diabetes (T2D) detected by an oral glucose tolerance test, according to ADA guidelines; history or clinical evidence of previous cardiovascular events, administration of any drugs interfering with glucose metabolism or renal function; kidney, thyroid, endocrine and advanced liver diseases, transplanted patients, history of malignant disease; history of chronic abuse of alcohol ( $>40 \mathrm{~g} / \mathrm{die}$ ) or recreational drugs. None of $\mathrm{HCV}$ patients had liver cirrhosis, detected by clinical and ultrasound examinations. In addition, in these patients we excluded membranoproliferative glomerulonephritis by $24-\mathrm{h}$ proteinuria and cryoglobulinemia.

We performed measurements of height and weight according to a standardized protocol, and body mass index (BMI) was calculated as kilograms per square meter. The Ethics Committee approved the protocol and informed written consent was obtained from all participants. All the investigations were performed in accordance with the principles of the Declaration of Helsinki.

\section{Blood pressure measurements}

Readings of clinic blood pressure (BP) were obtained in the left arm of the supine patients, after 5 min of quiet rest, with a mercury sphygmomanometer. A minimum of three $\mathrm{BP}$ readings were taken on three separate occasions at least 2 weeks apart. Systolic and diastolic BP were recorded at the first appearance (phase I) and the disappearance (phase V) of Korotkoff sounds. Baseline BP values were the average of the last two of the three consecutive measurements obtained at intervals of $3 \mathrm{~min}$. Patients with a clinic systolic BP (SBP) $<130 \mathrm{mmHg}$ and diastolic BP (DBP) $<80 \mathrm{mmHg}$ were defined as normotensives.

\section{Laboratory determinations}

All laboratory measurements were performed after $12 \mathrm{~h}$ of fasting. Plasma glucose was determined immediately by the glucose oxidation method [Glucose analyzer, Beckman Coulter, Milan; intra-assay coefficient of variation $(\mathrm{CV})$ $2.2 \%$, inter-assay CV $3.8 \%$ ]. Serum insulin was determined in duplicate by a highly specific radioimmunoassay using two monoclonal antibodies; intra-assay CV $2.1 \%$, inter-assay CV $2.9 \%$. Total, low-density lipoprotein(LDL), and high-density lipoprotein- (HDL) cholesterol and triglyceride concentrations were measured by enzymatic methods (Roche Diagnostics GmbH, Mannheim, Germany). Quantitative HCV-RNA was assayed by a realtime polymerase chain reaction assay.

\section{Insulin resistance evaluation}

IR was estimated by homeostasis model assessment (HOMA) according to the following equation: $\mathrm{HOMA}=$ insulin $(\mu \mathrm{U} / \mathrm{ml} \times$ glucose $(\mathrm{mmol} / \mathrm{l})] / 22.5$ [19]

\section{Renal function evaluation}

Creatinine was measured by using Jaffe methodology. Values of estimated glomerular filtration rate (e-GFR, mL/ $\min / 1.73 \mathrm{~m}^{2}$ ) were calculated by using the equation proposed by investigators in the chronic kidney disease epidemiology (CKD-EPI) collaboration [20].

\section{Statistical analysis}

ANOVA for continuous clinical and biological data was performed to test the differences among groups, and the Bonferroni post hoc test for multiple comparisons was further performed; for dichotomic variables we used the $X^{2}$ test. Data are expressed as mean $\pm \mathrm{SD}$, and binary data as percent frequency. Linear regression analysis was performed to correlate e-GFR with the following covariates: 
age, BMI, waist, SBP, DBP, pulse pressure (PP), LDL- and HDL-cholesterol, triglyceride, fasting plasma glucose and insulin, HOMA and uric acid. Subsequently, to define the independent predictors of renal function, variables reaching statistical significance, were inserted in a stepwise multivariate linear regression model. In this one, to avoid a possible colinearity, we did not include age, fasting glucose and insulin, but we considered only HOMA. Correlational analysis was performed in the whole study population and in the three groups separately. Differences were assumed to be significant at $P<0.05$. All calculations were done with a standard statistical package (SPSS for Windows version 20.0, Chicago, IL, USA).

\section{Results}

\section{Study population}

Clinical and laboratory characteristics of the study population are reported in Table 1. There were no significant differences among groups for age, gender, smoking and heart rate. Of interest, $\mathrm{HCV}^{+}$patients, with respect to both healthy and MS subjects show a lower and significant $(P<0.0001)$ e-GFR (Fig. 1). In addition, regarding other biochemical variables, $\mathrm{HCV}^{+}$patients, in comparison with healthy subjects, have higher triglyceride level, creatinine, fasting insulin and HOMA, and lower BMI, total and HDLcholesterol.

On the contrary, $\mathrm{HCV}^{+}$patients in comparison with the MS group have a lower BMI, waist circumference, systolic and diastolic BP, cholesterol, triglyceride, and fasting glucose. Of interest, no significant differences are found in fasting insulin and HOMA values $(3.7 \pm 1.4$ vs $3.4 \pm 1.4$; $P=0.287$ ).

In $\mathrm{HCV}^{+}$patients the mean value of HCV-RNA was $3579 \pm 1710 \times 10^{3}(\mathrm{UI} / \mathrm{ml})$.

\section{Correlational analysis}

A linear regression analysis was performed to test the correlation between e-GFR and different covariates in the whole study population and in the three groups separately (Table 2). In the whole study population, e-GFR is significantly correlated with HDL-cholesterol and inversely with age, waist circumference, SBP, DBP, PP, triglyceride, fasting glucose, insulin, HOMA and uric acid. In the MS and $\mathrm{HCV}^{+}$groups, e-GFR is statistically correlated with age, waist circumference, HDL cholesterol, uric acid, fasting glucose, insulin and HOMA. In addition, in the MS group, the other covariates that correlate with e-GFR are: SBP and pulse pressure.
A stepwise multivariate linear regression analysis was performed to evaluate the independent predictors of e-GFR in the entire population and in the three different groups (Table 3). In the whole population, as well as in MS and $\mathrm{HCV}^{+}$groups, HOMA is the major predictor of e-GFR, accounting for $25.8,30.8$ and $27.8 \%$ of its variation, respectively. Of interest, 1 unit of HOMA increase produces a reduction of $-6.062 \mathrm{ml} / \mathrm{min} / 1.73 \mathrm{~m}^{2}$ in MS group and of $-3.155 \mathrm{ml} / \mathrm{min} / 1.73 \mathrm{~m}^{2}$ in $\mathrm{HCV}^{+}$patients. In the whole population, other independent predictors of e-GFR are HDL cholesterol, uric acid and waist circumference, accounting for, respectively, another 9.4, 3.5 and $1.2 \%$ of its variation. In the MS group, the other covariates retained in the model are uric acid, waist circumference, gender and HDL cholesterol accounting for, respectively, 10.3, 5.4, 4.3 and $2.4 \%$ of e-GFR variation. In the $\mathrm{HCV}^{+}$group, covariates retained in the final model are gender, waist circumference, uric acid and HDL cholesterol accounting for, respectively, another 11.6, 10.5, 2.6 and $1.6 \%$ of its variation.

\section{Discussion}

The results of this study demonstrate that $\mathrm{HCV}^{+}$patients, in comparison with healthy subjects and MS patients, have a significant decrease in e-GFR. These results have a clinical relevance because they help to clarify the pathogenetic role of $\mathrm{HCV}$ infection in promoting renal damage in a group of newly diagnosed $\mathrm{HCV}^{+}$patients without membranoproliferative glomerulonephritis or cryoglobulinemia, all established factors affecting renal function in this clinical setting. Contrary to other previously published studies, aimed at demonstrating an association between $\mathrm{HCV}$ infection and CKD, our data emphasize the early role of viral infection on renal function decrease, also in patients with still preserved renal function; this condition has a clinical relevance because it should alert physicians to routinely evaluate renal function in these patients [13$16,21,22]$. Thus, on the basis of this evidence, we hypothesize that early detection of a loss of renal function may be useful in slowing the progression to CKD by appropriate therapeutic interventions. The large sample size and detailed characterization of patients confer to our study a very important clinical and biological plausibility that contributes to clarify the association between $\mathrm{HCV}$ infection and reduction of renal function, similar to that observed for other subclinical organ damages [5, 6].

Another important finding obtained by our study consists in the fact that IR is a primary and independent predictor of e-GFR variation in both $\mathrm{HCV}^{+}$and MS patients, as already reported [11, 12]. The interaction between metabolic and hemodynamic parameters may be explained 


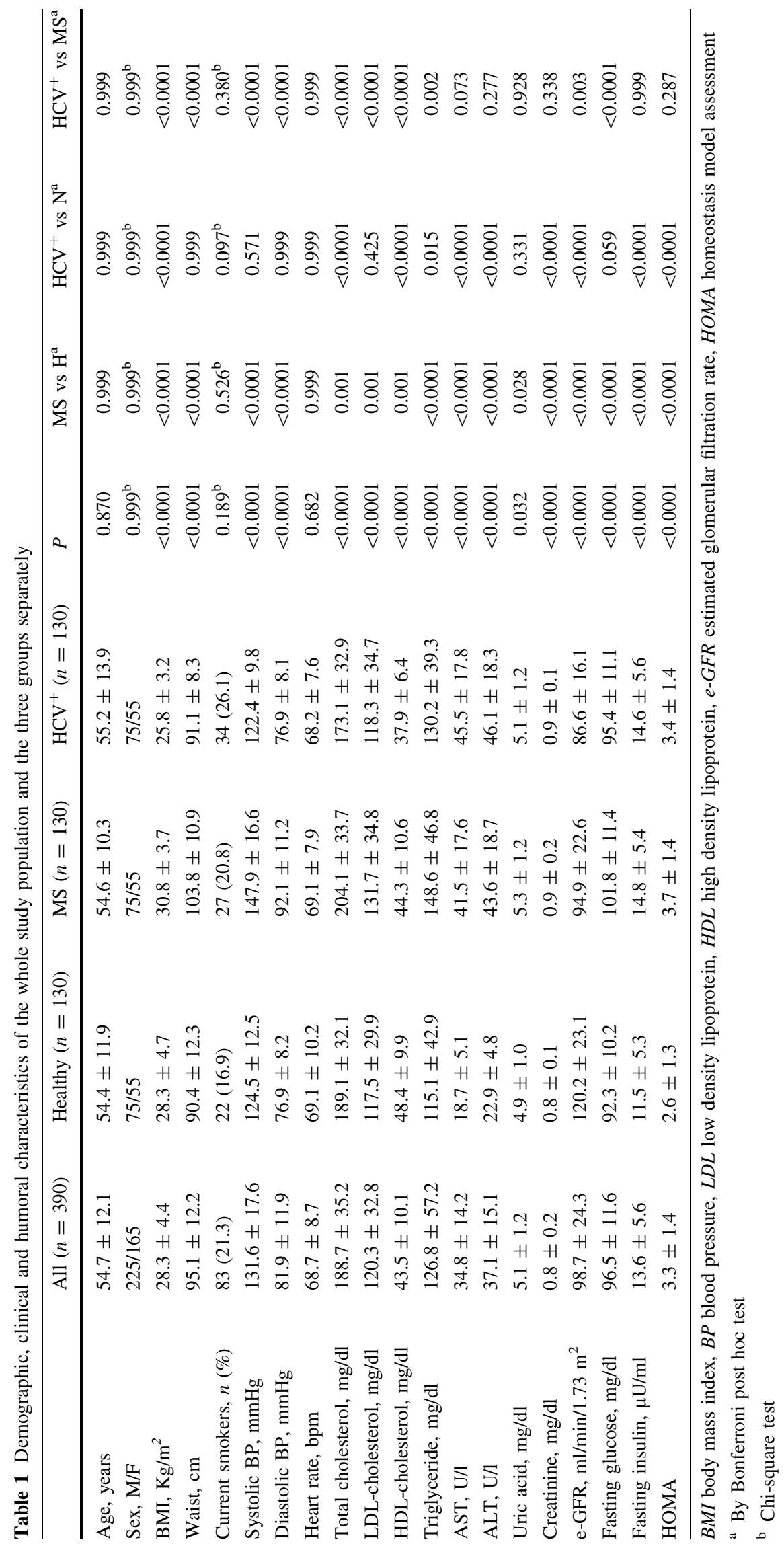




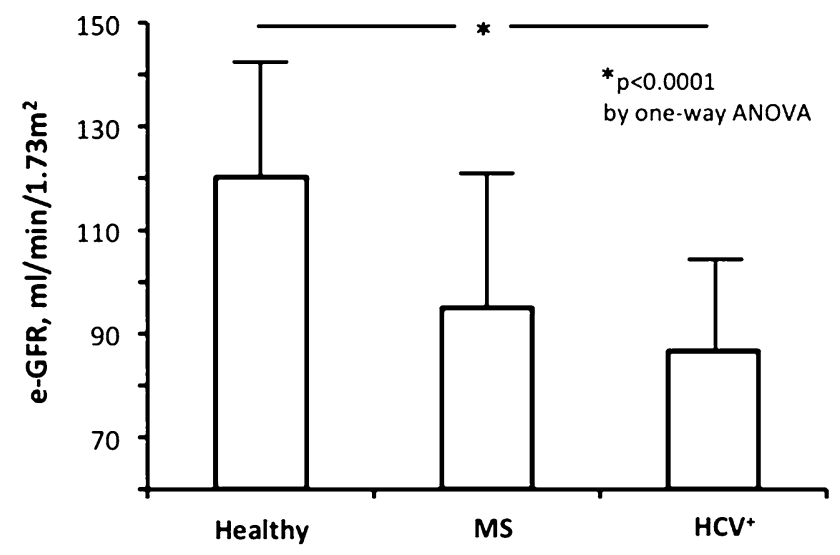

Fig. 1 We graphically reported estimated glomerular filtration rate (e-GFR) mean values in all three different study groups. $\mathrm{HCV}^{+}$ patients presented a significantly lower e-GFR in comparison with both healthy subjects and metabolic syndrome (MS) patients $(P<0.0001$, by ANOVA $)$

by previously published data demonstrating that functional GFR reduction is inversely related to circulating IGF-1 levels [23]. In fact, there is evidence that low IGF-1 levels impair vasodilating properties of renal arterioles that decrease both renal blood flow and GFR. This evidence is explained by our and other data demonstrating that a dynamic balance exists between IR-related hyperinsulinemia and IGF-1 levels [24-26]. In accordance with this, it is clearly demonstrated that endogenous IGF-1 levels improve GFR by stimulating the glomerular L-argininenitric oxide pathway, without any systemic hemodynamic changes [27, 28].

It is well established that visceral obesity is characterized by an IR status; in fact, there is growing evidence demonstrating that a selective excess of visceral adipose tissue is associated with a higher risk to develop IR because it acts as an endocrine organ that produces several substances involved in the regulation of metabolic, inflammatory and immune responses [29, 30]. In keeping with this, it is not surprising that MS patients develop an IR that may be considered the common soil for metabolic and hemodynamic alterations that produce the definition of this syndrome, and that may promote the associated target organ damage. In accordance with this, the presence of a MS entails a twofold increased risk for microalbuminuria, an early indicator of renal damage [31]. In $\mathrm{HCV}^{+}$patients, it has been demonstrated that the IR degree may be explained through a direct interaction between viral products and the insulin signaling pathway via IRS-1-PI3-kinase-Akt [32-34]. The biological plausibility of this finding is also supported by the present results demonstrating that HOMA is the major determinant of e-GFR decrease in $\mathrm{MS}$ and $\mathrm{HCV}^{+}$patients, accounting for 30.8 and $27.8 \%$ of its variation, respectively. In addition, $\mathrm{HCV}^{+}$patients, in comparison with healthy subjects, while presenting a significant lower BMI, show an increased, although not significant, waist circumference. This is the second independent predictor of e-GFR accounting for $10.5 \%$ of its variation.

Interestingly, all these conditions interact between them in promoting the e-GFR reduction with important clinical implications, considering that the loss of renal function is an independent predictor of cardiovascular morbidity and mortality $[9,10]$. For these reasons CKD is a relevant health problem not only in the specific setting of patients but also in the general population..

Table 2 Linear regression analysis between e-GFR and different covariates in the whole study population and in the three groups

\begin{tabular}{|c|c|c|c|c|c|c|c|c|}
\hline & \multicolumn{2}{|c|}{ All $(n=390)$} & \multicolumn{2}{|c|}{ Healthy $(n=130)$} & \multicolumn{2}{|c|}{$\operatorname{MS}(n=130)$} & \multicolumn{2}{|c|}{$\mathrm{HCV}^{+}(n=130)$} \\
\hline & $r$ & $P$ & $r$ & $P$ & $r$ & $P$ & $r$ & $P$ \\
\hline Age, years & -0.265 & $<0.0001$ & -0.257 & 0.002 & -0.196 & 0.013 & -0.516 & $<0.0001$ \\
\hline BMI, $\mathrm{kg} / \mathrm{m}^{2}$ & 0.038 & 0.230 & -0.055 & 0.267 & -0.027 & 0.379 & 0.008 & 0.465 \\
\hline Waist, cm & -0.283 & $<0.0001$ & -0.147 & 0.048 & -0.351 & $<0.0001$ & -0.380 & $<0.0001$ \\
\hline Systolic BP, mmHg & -0.167 & $<0.0001$ & -0.0201 & 0.410 & -0.328 & $<0.0001$ & 0.043 & 0.313 \\
\hline Diastolic BP, mmHg & -0.094 & 0.032 & -0.014 & 0.438 & 0.037 & 0.336 & -0.033 & 0.356 \\
\hline $\mathrm{PP}, \mathrm{mmHg}$ & -0.146 & 0.002 & -0.013 & 0.440 & -0.389 & $<0.0001$ & 0.080 & 0.183 \\
\hline LDL Cholesterol, mg/dl & -0.081 & 0.055 & -0.342 & $<0.0001$ & -0.080 & 0.182 & 0.090 & 0.153 \\
\hline HDL Cholesterol, mg/dl & 0.433 & $<0.0001$ & 0.144 & 0.052 & 0.384 & $<0.0001$ & 0.341 & $<0.0001$ \\
\hline Triglyceride, mg/dl & -0.137 & 0.003 & -0.099 & 0.132 & -0.001 & 0.494 & 0.049 & 0.289 \\
\hline Fasting glucose, mg/dl & -0.294 & $<0.0001$ & -0.119 & 0.089 & -0.279 & 0.001 & -0.340 & $<0.0001$ \\
\hline Fasting insulin, $\mu \mathrm{UI} / \mathrm{ml}$ & -0.468 & $<0.0001$ & -0.259 & 0.001 & -0.514 & $<0.0001$ & -0.472 & $<0.0001$ \\
\hline HOMA & -0.508 & $<0.0001$ & -0.273 & 0.001 & -0.555 & $<0.0001$ & -0.527 & $<0.0001$ \\
\hline Uric acid, $\mathrm{mg} / \mathrm{dl}$ & -0.360 & $<0.0001$ & -0.266 & 0.001 & -0.444 & $<0.0001$ & -0.407 & $<0.0001$ \\
\hline
\end{tabular}

$M S$ metabolic syndrome, $B M I$ body mass index, $B P$ blood pressure, $P P$ pulse pressure, $L D L$ low density lipoprotein, $H D L$ high density lipoprotein, $e-G F R$ estimated glomerular filtration rate, HOMA homeostasis model assessment 
Table 3 Stepwise multiple regression analysis on e-GFR, as dependent variable in whole study population and in the three groups (not including age, fasting glucose and insulin, pulse pressure when significant to avoid colinearity)

\begin{tabular}{|c|c|c|c|c|c|c|c|c|}
\hline & \multicolumn{2}{|l|}{ All $(n=390)$} & \multicolumn{2}{|c|}{ Healthy $(n=130)$} & \multicolumn{2}{|l|}{$\operatorname{MS}(n=130)$} & \multicolumn{2}{|c|}{$\mathrm{HCV}^{+}(n=130)$} \\
\hline & Partial $R^{2}(\%)$ & $P$ & Partial $R^{2}(\%)$ & $P$ & Partial $R^{2}(\%)$ & $P$ & Partial $R^{2}(\%)$ & $P$ \\
\hline HOMA & 25.8 & $<0.0001$ & 6.5 & 0.001 & 30.8 & $<0.0001$ & 27.8 & $<0.0001$ \\
\hline HDL cholesterol, mg/dl & 9.4 & $<0.0001$ & - & - & 2.5 & 0.011 & 1.6 & 0.037 \\
\hline Uric acid, $\mathrm{mg} / \mathrm{dl}$ & 3.5 & $<0.0001$ & 2.2 & 0.049 & 10.3 & $<0.0001$ & 2.6 & 0.010 \\
\hline Waist, cm & 1.2 & 0.005 & - & - & 5.4 & $<0.0001$ & 10.5 & $<0.0001$ \\
\hline LDL cholesterol, mg/dl & - & - & 8.7 & $<0.0001$ & - & - & - & - \\
\hline Gender, male/female & - & - & 12.4 & $<0.0001$ & 4.0 & 0.002 & 11.6 & $<0.0001$ \\
\hline Smoking, yes/no & - & - & 2.5 & 0.035 & - & - & - & - \\
\hline Total $R^{2}(\%)$ & 39.9 & - & 32.3 & - & 51.0 & - & 54.1 & - \\
\hline
\end{tabular}

$M S$ metabolic syndrome, $e$-GFR estimated glomerular filtration rate, HOMA homeostasis model assessment

\section{Study limitation}

These data are associative, and do not definitively support regarding the role of metabolic alterations in promoting renal dysfunction.

Recently, new drugs are reported to be able to counteract $\mathrm{HCV}$ replication and limit the progression of liver disease with a high likelihood of healing. However, at this moment, no data are available regarding the impact of microbiological healing on extrahepatic clinical manifestations, which contribute to morbidity and mortality in these patients.

\section{Compliance with ethical standards}

Funding No relationship with any industry or financial support exists.

Conflict of interest The authors declare that they have no conflict of interest.

Statement of human and animal rights This article does not contain any studies with human participants or animals performed by any of the authors.

Informed consent Written informed consent was obtained from all participants.

\section{References}

1. Butt AA, Xiaoqiang W, Budoff M, Leaf D, Kuller LH, Justice AC (2009) Hepatitis C virus infection and the risk of coronary disease. Clin Infect Dis 49:225-232

2. Völzke H, Schwahn C, Wolff B et al (2004) Hepatitis B and C virus infection and the risk of atherosclerosis in a general population. Atherosclerosis 174:99-103

3. Targher G, Bertolini L, Padovani R, Rodella S, Arcaro G, Day C (2007) Differences and similarities in early atherosclerosis between patients with non-alcoholic steatohepatitis and chronic hepatitis B and C. J Hepatol 46:1126-1132

4. Mostafa A, Mohamed MK, Saeed M, Hasan A, Fontanet A, Godsland I, Coady E, Esmat G, El-Hoseiny M, Abdul-Hamid M,
Hughes A, Chaturvedi N (2010) Hepatitis C infection and clearance: impact on atherosclerosis and cardiometabolic risk factors. Gut 59:1135-1140

5. Perticone M, Miceli S, Maio R, Caroleo B, Sciacqua A, Tassone EJ, Greco L, Staltari O, Sesti G, Perticone F (2014) Chronic HCV infection increases cardiac left ventricular mass index in normotensive patients. J Hepatol 61:755-760

6. Perticone M, Maio R, Tassone EJ, Tripepi G, Di Cello S, Miceli S, Caroleo B, Sciacqua A, Licata A, Sesti G, Perticone F (2015) Insulin-resistance $\mathrm{HCV}$ infection-related affects vascular stiffness in normotensives. Atherosclerosis 238:108-112

7. Schillaci G, Verdecchia P, Porcellati C, Cuccurullo O, Cosco C, Perticone F (2000) Continuous relation between left ventricular mass and cardiovascular risk in essential hypertension. Hypertension 35:580-586

8. Willum-Hansen T, Staessen JA, Torp-Pedersen C, Rasmussen S, Thijs L, Ibsen H, Jeppesen J (2006) Prognostic value of aortic pulse wave velocity as index of arterial stiffness in the general population. Circulation 113:664-670

9. Go AS, Chertow GM, Fan D, McCulloch CE, Hsu CY (2004) Chronic kidney disease and the risks of death, cardiovascular events, and hospitalization. N Engl J Med 351:1296-1305 (Erratum in: (2008) N Engl J Med 18:4)

10. Fried LF, Shlipak MG, Crump C, Bleyer AJ, Gottdiener JS, Kronmal RA, Kuller LH, Newman AB (2003) Renal insufficiency as predictor of cardiovascular outcomes and mortality in elderly individuals. J Am Coll Cardiol 41:1364-1372

11. Chen J, Muntner P, Hamm LL, Jones DW, Batuman V, Fonseca V, Whelton PK, He J (2004) The metabolic Syndrome and chronic renal disease in US adults. Ann Intern Med 140:167-174

12. Kurella M, Lo JC, Chertow GM (2005) Metabolic syndrome and the risk for chronic Kidney disease among nondiabetic adults. J Am Soc Nephrol 16:2134-2140

13. Asrani SK, Buchanan P, Pinsky B, Rey LR, Schnitzler M, Kanwal F (2010) Lack of association between hepatitis C infection and chronic kidney disease. Clin Gastroenterol Hepatol 8:79-84

14. Moe SM, Pampalone AJ, Ofner S, Rosenman M, Teal E, Hui SL (2008) Association of hepatitis $C$ virus infection with prevalence and development of kidney disease. Am J Kidney Dis 51:885-892

15. Tsui JI, Vittinghoff E, Shlipak MG, O'Hare AM (2006) Relationship between hepatitis $\mathrm{C}$ and chronic kidney disease: results from the Third National Health and Nutrition Examination Survey. J Am Soc Nephrol 17:1168-1174

16. Fabrizi F, Martin P, Dixit V, Messa P (2012) Hepatitis C virus infection and kidney disease: a meta-analysis. Clin J Am Soc Nephrol 7:549-557 
17. Succurro E, Arturi F, Lugarà M, Grembiale A, Fiorentino TV, Caruso V, Andreozzi F, Sciacqua A, Hribal ML, Perticone F, Sesti G (2010) One-hour postload plasma glucose levels are associated with kidney dysfunction. Clin J Am Soc Nephrol 5:1922-1927

18. Alberti KG, Eckel RH, Grundy SM et al (2009) Harmonizing the metabolic syndrome: a joint interim statement of the International Diabetes Federation Task Force on Epidemiology and Prevention; National Heart, Lung, and Blood Institute; American Heart Association; World Heart Federation; International Atherosclerosis Society; and International Association for the Study of Obesity. Circulation 120:1640-1645

19. Matthews DR, Hosker JP, Rudenski AS, Naylor BA, Treacher DF, Turner RC (1985) Homeostasis model assessment: insulin resistance and beta-cell function from fasting plasma glucose and insulin concentrations in man. Diabetologia 28:412-419

20. Levey AS, Stevens LA, Schmid CH, Zhang YL, Castro AF 3rd, Feldman HI, Kusek JW, Eggers P, Van Lente F, Greene T, Coresh J, CKD-EPI (chronic kidney disease epidemiology collaboration) (2009) A new equation to estimate glomerular filtration rate. Ann Intern Med 150:604-612

21. Satapathy SK, Lingisetty CS, Williams S (2012) Higher prevalence of chronic kidney disease and shorter renal survival in patients with chronic hepatitis $\mathrm{C}$ virus infection. Hepatol Int 6:369-378

22. Butt AA, Wang X, Fried LF (2011) HCV infection and the incidence of CKD. Am J Kidney Dis 57:396-402

23. Perticone F, Maio R, Sciacqua A, Perticone M, Laino I, Miceli S, Mazzaferro D, Pascale A, Andreozzi F, Sesti G (2009) Insulinlike growth factor-1 and glomerular filtration rate in hypertensive patients. J Hypertens 27:613-617

24. Sesti G, Sciacqua A, Cardellini M, Marini MA, Maio R, Vatrano M, Succurro E, Lauro R, Federici M, Perticone F (2005) Plasma concentration of IGF-I is independently associated with insulin sensitivity in subjects with different degrees of glucose tolerance. Diabetes Care 28:120-125
25. Thrailkill KM, Quattrin T, Baker L, Kuntze JE, Compton PG, Martha PM Jr (1999) Cotherapy with recombinant human insulinlike growth factor I and insulin improves glycemic control in type 1 diabetes: RhIGF-I in IDDM Study Group. Diabetes Care 22:585-592

26. Sandhu MS, Heald AH, Gibson JM, Cruickshank JK, Dunger DB, Wareham NJ (2002) Circulating concentrations of insulin like growth factor-I and development of glucose intolerance: a prospective observational study. Lancet 359:1740-1745

27. Haylor J, Singh I, el Nahas AM (1991) Nitric oxide synthesis inhibitor prevents vasodilation by insulin-like growth factor I. Kidney Int 39:333-335

28. Vijayan A, Franklin SC, Behrend T, Hammerman MR, Miller SB (1999) Insulin-like growth factor 1 improves renal function in patients with end-stage chronic renal failure. Am J Physiol 276:R929-R934

29. Shoelson SE, Herrero L, Naaz A (2005) Obesity, inflammation, and insulin resistance. Gastroenterology 128:636-641

30. Despres JP, Lemieux I (2006) Abdominal obesity and metabolic syndrome. Nature 444:881-887

31. Leoncini G, Ratto E, Viazzi F, Vaccaro V, Parodi D, Parodi A, Falqui V, Tomolillo C, Deferrari G, Pontremoli R (2005) Metabolic syndrome is associated with early signs of organ damage in nondiabetic, hypertensive patients. J Intern Med 257:454-460

32. Shintani Y, Fujie H, Miyoshi H, Tsutsumi T, Tsukamoto K, Kimura S, Moriya K, Koike K (2004) Hepatitis C virus infection and diabetes: direct involvement of the virus in the development of insulin resistance. Gastroenterology 126:840-848

33. Bugianesi E, Salamone F, Negro F (2012) The interaction of metabolic factors with $\mathrm{HCV}$ infection: does it matter? J Hepatol 56(suppl 1):S56-S65

34. Biddinger SB, Kahn CR (2006) From mice to men: insights into the insulin resistance syndromes. Ann Rev Physiol 68:123-158 